\begin{abstract}
Iranica
Abstracta Iranica Revue bibliographique pour le domaine irano-aryen

Volume 37-38-39 | 2018

Comptes rendus des publications de 2014-2016
\end{abstract}

\title{
Yuka Kadoi (dir.). Arthur Upham Pope and A New Survey of Persian Art
}

\section{Sarah Piram}

\section{(2) OpenEdition \\ 1 Journals}

\section{Édition électronique}

URL : http://journals.openedition.org/abstractairanica/42714

DOI : 10.4000/abstractairanica.42714

ISBN : 1961-960X

ISSN : 1961-960X

Éditeur :

CNRS (UMR 7528 Mondes iraniens et indiens), Éditions de l'IFRI

Référence électronique

Sarah Piram, " Yuka Kadoi (dir.). Arthur Upham Pope and A New Survey of Persian Art », Abstracta Iranica [En ligne], Volume 37-38-39 | 2018, document 8, mis en ligne le 10 mars 2018, consulté le 28 septembre 2020. URL : http://journals.openedition.org/abstractairanica/42714 ; DOI : https://doi.org/ 10.4000/abstractairanica.42714

Ce document a été généré automatiquement le 28 septembre 2020

Tous droits réservés 


\title{
Yuka Kadoi (dir.). Arthur Upham Pope and A New Survey of Persian Art
}

\author{
Sarah Piram
}

\section{RÉFÉRENCE}

Yuka Kadoi (dir.), Arthur Upham Pope and A New Survey of Persian Art, Brill, Leyde-

Boston, 2016, 417 p., ISBN: 978-9004-30989-0

1 Cet ouvrage, dirigé par Yuka Kadoi, est le fruit d'un symposium international qui s'est tenu à l'Art Institute de Chicago les 9 et 10 septembre 2010. Arthur Upham Pope and A New Survey of Persian Art met en lumière le rôle d'Arthur Upham Pope (1881-1969), l'un des pionniers de l'étude et de la connaissance du patrimoine iranien dans la première moitié du $\mathrm{XX}^{\mathrm{e}}$ siècle. Célèbre pour son incontournable Survey of Persian Art. From Prehistoric Times to the Present (Oxford University Press, publié en six volumes entre 1938 et 1939), cet orientaliste américain a consacré sa carrière à l'Iran, en organisant différents congrès internationaux et en valorisant le patrimoine iranien dans les musées occidentaux, principalement aux États-Unis. L'ouvrage retrace notamment l'influence de Pope dans la montée du collectionnisme de l'art iranien entre la fin des années 1920 et jusqu'aux années 1930.

Grâce à l'étude de ses archives, il est possible de mieux comprendre le rôle qu'a suscité ce chercheur américain dans la connaissance de l'Iran avant et - surtout - après l'avènement de l'Islam. Dans une longue introduction, Kadoi revient sur la notion même d'art persan remise en question dès la fin du XIX ${ }^{e}$ siècle, et surtout au tournant des années 1930, avec la montée d'une conscience patrimoniale d'envergure en Iran dont Pope en a été un des déclencheurs. Figure controversée pour son implication dans le marché de l'art, mais toujours respectée pour son dévouement à l'Iran, Pope est présenté ici comme un pionnier de l'étude de l'art persan. Kadoi propose de s'attacher à 
ses différentes réalisations professionnelles en les plaçant dans un cadre historique et culturel propre à la première moitié du $\mathrm{XX}^{\mathrm{e}}$ siècle.

3 L'ouvrage propose ainsi de se pencher sur les différentes facettes de la figure emblématique de Pope, à travers quatorze contributions divisées en cinq parties :

La première partie réunit deux articles portant sur Pope, Ackerman et leurs contemporains : Robert Hillenbrand signe ainsi un article intitulé : «The Scramble for Persian Art: Pope and His Rivals» (p. 15-45), suivi de Talinn Grigor « Gendered Politics of Persian Art: Pope and His Partner » (p. 47-73).

5 La deuxième partie est biographique, elle se décline à travers trois contributions: "Arthur Upham Pope: His Life and Times» par Jonathan M. Bloom (p. 78-95), "Archaeology in Iran and the Experience of Arthur Upham Pope " par Donald Whitcomb (p. 98-109), et "Arthur Upham Pope and the Study of Persian Islamic Architecture » par Bernard O’Kane (p. 111-124).

6 La troisième partie est consacrée à la question de la conservation, du collectionnisme et du marché de l'art en lien avec l'art de l'Iran préislamique. L'article de Lindsay Allen a pour titre " 'The Greatest Enterprise': Arthur Upham Pope, Persepolis and Achaemenid Antiquities » (p. 127-167), vient ensuite " Arthur Upham Pope and the Sassanians » par Judith A. Lerner (p. 169-232).

7 La quatrième partie se penche sur la même question, mais concerne exclusivement l'art de l'Iran islamique. Cette section rassemble le plus grand nombre de contributions: "The Rise of Persian Art Connoisseurship: Arthur Upham Pope and Early TwentiethCentury Chicago » par Yuka Kadoi (p. 235-265), «Arthur Upham Pope and Collecting Persian Art for Kansas City » par Kimberley Masteller (p. 267-289), « Equivocal Position as Expert or Dealer! The Long and Contentious Relationship of George Hewitt Myers and Arthur Upham Pope » par Sumru Belger Krody (p. 291-307), « My Dear Holmes: Arthur Upham Pope and the Museum of Fine Arts, Boston » par Laura Weinstein (p. 309-325), « Filming, Photographing and Purveying in 'The New Iran': The Legacy of Stephen H. Nyman, ca. 1937-42 » par Keelan Overton (p. 327-370).

8 Enfin, la dernière partie de ce livre ouvre une perspective intéressante sur l'héritage d'Arthur Upham Pope : «Surveying Persian Art in Light of A Survey of Persian Art » par Sheila S. Blair (p. 373-395), «Arthur Upham Pope: A Personal Memoir » par Cornelia Montgomery (p. 397-411).

L'ouvrage compte au total quatre-vingt-six illustrations, en noir et blanc pour les images d'archives et en couleur pour les photographies les plus récentes. En plus d'offrir un panorama des actions de Pope dans l'étude de l'histoire de l'art iranien, le livre donne des perspectives nouvelles sur l'historiographie de l'art islamique qui, en s'étant développé dans les années 1990, connaît un certain renouvellement depuis quelques années, à travers l'étude de personnalités majeures comme Ernst Herzfeld ou André Godard. 


\section{AUTEURS}

SARAH PIRAM

Doctorante en histoire de l'art contemporain, Université Paris-Nanterre 